\title{
On the Communication Requirements of Verifying the VCG Outcome
}

\section{Citation}

Lahaie, Sébastien and David C. Parkes. 2008. On the communication requirements of verifying the VCG outcome. In Proceedings of the 9th ACM Conference on Electronic Commerce: July 8-12, 2008, Chicago, Ill., ed. J. Riedl; T. Sandholm, 78-87. New York, N.Y.: ACM Press.

\section{Published Version}

http://doi.acm.org/10.1145/1386790.1386806

\section{Permanent link}

http://nrs.harvard.edu/urn-3:HUL.InstRepos:4039779

\section{Terms of Use}

This article was downloaded from Harvard University's DASH repository, and is made available under the terms and conditions applicable to Other Posted Material, as set forth at http:// nrs.harvard.edu/urn-3:HUL.InstRepos:dash.current.terms-of-use\#LAA

\section{Share Your Story}

The Harvard community has made this article openly available.

Please share how this access benefits you. Submit a story.

Accessibility 


\section{On the Communication Requirements of Verifying the VCG Outcome}

\author{
Sébastien Lahaie \\ Yahoo Research \\ New York, NY 10018 \\ lahaies@yahoo-inc.com
}

\author{
David C. Parkes \\ School of Engineering and Applied Sciences \\ Harvard University \\ Cambridge, MA 02138 \\ parkes@eecs.harvard.edu
}

\begin{abstract}
We consider the amount of communication required to verify the outcome of the Vickrey-Clarke-Groves (VCG) mechanism: an efficient allocation together with incentivizing VCG payments. We compare this to the communication required to verify the efficient decision rule alone, to assess the overhead imposed by VCG payments. Our characterizations are obtained by leveraging a connection between the VCG outcome and a price equilibrium concept known as universal competitive equilibrium. We consider four related environments within a common framework: the classic single-item setting, the multi-unit setting with decreasing marginal values, the classic assignment problem with unitdemand valuations, and the multi-unit assignment problem with substitutes valuations. We find that the single-unit settings have zero overhead, whereas the multi-unit settings can have significant positive overhead. With multiple units, the naïve VCG protocol that runs several efficient protocols in sequence (one with all agents, and ones with an agent removed, for each agent) is asymptotically optimal for several parameter settings of the number of agents, commodities, and units.
\end{abstract}

\section{Categories and Subject Descriptors}

F.2 [Theory of Computation]: Analysis of Algorithms and Problem Complexity; J.4 [Computer Applications]: Social and Behavioral Sciences-Economics

\section{General Terms}

Economics, Theory

\section{INTRODUCTION}

The Vickrey-Clarke-Groves (VCG) mechanism holds a central place in the theory of mechanism design due to its unique efficiency, incentive, and revenue properties. In its traditional formulation, the VCG mechanism is "direct": the agents reveal their complete preferences to a center who then

Permission to make digital or hard copies of all or part of this work for personal or classroom use is granted without fee provided that copies are not made or distributed for profit or commercial advantage and that copies bear this notice and the full citation on the first page. To copy otherwise, to republish, to post on servers or to redistribute to lists, requires prior specific permission and/or a fee.

EC'08, July 8-12, 2008, Chicago, Illinois, USA.

Copyright 2008 ACM 978-1-60558-169-9/08/07 ...\$5.00. implements an efficient outcome. The center also charges payments that make it a dominant strategy for agents to be truthful. When incentives are the only concern, the revelation principle states that there is no loss of generality in restricting attention to direct mechanisms. However, other considerations such as privacy often make "indirect" mechanisms more attractive in practice [28].

Several indirect mechanisms have been proposed that compute the VCG outcome (allocation and payments) for various environments. These take the form of iterative auctions $[1,2,7,22]$. In these cases VCG payments bring sincere bidding (i.e., price-taking behavior) into an ex post Nash equilibrium. Among other features, these auctions offer the possibility that agent preferences need not be fully revealed before the desired outcome is reached. This connects with a second theme of the literature on resource allocation mechanisms: communication [14, 23].

In this paper we analyze how much communication is required to realize the VCG outcome. The incentivizing transfers defined by the VCG mechanism impose an additional "overhead" on the communication requirements of the efficient decision rule alone. We consider this overhead for several environments: the classic single-item setting, the multi-unit setting with decreasing marginal values, the classic assignment problem with unit-demand valuations, and the multi-unit assignment problem with substitutes valuations.

We consider the "nondeterministic" problem of verifying that an allocation-payment outcome is indeed a VCG outcome, rather than the more challenging "deterministic" problem of computing the outcome. Nonetheless, our results also have implications for deterministic communication. In characterizing communication requirements, we seek upper and lower bounds that match as closely as possible. Our lower bounds apply to deterministic communication as well, whereas our upper bounds are derived by proving the existence of price equilibria of certain dimensions. The latter can form the basis of formal "tâtonnement" procedures such as auctions; a typical approach is to use the existence of price equilibria to prove that certain linear programs compute an efficient, discrete allocation, and to then interpret dual methods (e.g., subgradient, primal-dual) on these programs as auction procedures [6].

Our key finding is that the single-unit settings impose no overhead, whereas in the multi-unit settings there can be a positive overhead, equivalent to a polynomial increase over the communication of efficient protocols that do not consider incentives. When our characterizations are not exact, they 
are still asymptotically tight for several parameter settings of the number of agents, commodities, and units.

Our analysis closely models that of Nisan and Segal [24] (henceforth NS) for the efficient decision rule. They first draw a connection between the communication requirements of efficient outcomes and of competitive equilibria, showing that they are equivalent. They then use this characterization to lower bound the communication requirements of efficiency for several valuation classes. In an analogous fashion, we leverage a connection between the VCG outcome and "universal competitive equilibria," introduced by Parkes and Ungar [26] (later subsumed by Mishra and Parkes [22]), to obtain lower bounds on communication for the various environments we consider. Our upper bounds are obtained through explicit constructions of universal competitive equilibrium prices.

To our knowledge, ours are the first lower bounds on the communication requirements of the VCG outcome in any nontrivial settings beyond the simple single-item auction. There are several results in the literature that show that linear prices do not suffice to guarantee the existence of a competitive or universal competitive equilibrium; see for example Gul and Stacchetti [12] and Mishra and Parkes [22]. Such results only show, using counterexamples, that some low-dimensional subspace of prices cannot encode the relevant equilibrium prices. This does not provide a lower bound on communication more generally, because special short encodings could be used for the constructed counterexamples. In contrast, we use a "fooling set" technique drawn from NS to show that no low-dimensional subspace can encode the relevant equilibrium prices, and this translates into lower bounds on communication.

Several mechanisms are incentive-compatible besides the VCG mechanism. Fadel and Segal [8] investigate the "communication cost of selfishness" more generally: what is the minimum possible overhead, over all incentive-compatible mechanisms, of computing incentivizing transfers? (They ask this question for deterministic communication, and consider other rules besides efficiency.) In the case of the efficient rule, Reichelstein [27] notes that giving each agent the sum of the others' utilities from the computed outcome gives incentivizing transfers - the efficient rule is implemented in ex post Nash equilibrium. Fadel and Segal therefore conclude that the overhead is at most the number of agents: the agents' utilities for the outcome can be appended to the communication of an efficient protocol, effectively communicating the transfers. Note though that this scheme can be extremely costly to implement, and is infeasible if the protocol is not allowed to run a deficit. The VCG mechanism remedies this problem, but at the cost of increased communication.

A similar approach can be used for the VCG outcome: we run an efficient protocol with all the agents, and then with each agent removed in turn. At the end of each run, the agents' utilities for the computed outcome are also communicated. This gives enough information to verify VCG payments besides the efficient outcome. Roughly, this multiplies the communication of the efficient protocol by the number of agents. One question we ask in this paper is whether there exist protocols with significantly smaller overhead than this "naïve" protocol. With multiple units of each commodity, the answer is negative for certain parameter settings: the naïve protocol is asymptotically optimal.
The key contributions of the paper are:

1. An almost exact characterization of the communication requirement of the VCG outcome when goods are homogeneous and agent valuations exhibit decreasing marginal values

2. An exact characterization of the communication requirement of the VCG outcome in the classic assignment problem with unit-demand valuations

3. A lower bound on the communication requirement of the VCG outcome in the multi-unit assignment problem with substitutes valuations, that is asymptotically tight for several parameter settings of the model

We believe the techniques used to construct upper and lower bounds for these various environments are also interesting in their own right.

Section 2 introduces the model, including the different valuation classes considered. Section 3 describes the efficient allocation problem, the VCG mechanism, and the communication model. Section 4 shows that verifying the VCG outcome is equivalent to verifying a universal competitive equilibrium. Section 5 describes the naïve protocol as well as its analog for verifying universal competitive equilibria, which gives a general bound on the overhead imposed by VCG payments. Section 6 then derives upper and lower bounds on the communication requirements of the VCG outcome for four different environments. Section 7 concludes.

\section{THE MODEL}

A seller wishes to allocate indivisible units of $m$ heterogeneous commodities among a set of $n$ agents. There are $l$ units of each commodity. Let $M$ be the set of commodities and $N=\{1, \ldots, n\}$ be the set of agents. Each agent's consumption set is $X=\left\{y \in \mathbf{Z}_{+}^{M}: y(j) \leq l, j \in M\right\}$, the set of bundles. ${ }^{1}$

Agent $i$ 's valuation $v_{i}$ assigns a real value to each bundle; it is his privately observed "type". Agent $i$ 's valuation is drawn from a class $\mathcal{V}_{i}$, and the set of possible valuation profiles, or environment, is the product set $\mathcal{V}=\times_{i \in N} \mathcal{V}_{i}$. We make the following assumptions on bidder values and utilities.

Private Values: Agent $i$ 's value for bundle $x_{i}, v_{i}\left(x_{i}\right)$, does not depend on the other agents' types.

Quasi-linearity: If agent $i$ obtains bundle $x_{i}$ and is charged $q_{i}$, it derives utility $v_{i}\left(x_{i}\right)-q_{i}$.

Monotonicity: If $x_{i}^{\prime} \geq x_{i}$, then $v_{i}\left(x_{i}^{\prime}\right) \geq v_{i}\left(x_{i}\right)$.

Concave-extensibility: The concave closure $\bar{v}_{i}$ of $v_{i}$ coincides with $v_{i}$ on $X^{2}{ }^{2}$

An agent's utility depends only on the bundle it receives and payment it issues, and not on allocations and other agents' transfers more generally.

${ }^{1}$ The notation $\mathbf{Z}_{+}^{M}$ denotes the set of vectors with components drawn from $\mathbf{Z}_{+}$(the non-negative integers) and indexed by elements of the set $M$. For $x_{i} \in \mathbf{Z}_{+}^{M}$, we write $x_{i}(j)$ to denote the component corresponding to $j \in M$.

${ }^{2}$ The concave closure $\bar{v}_{i}$ is the smallest concave function on $\left\{y \in \mathbf{R}_{+}^{M}: y(j) \leq l, j \in M\right\}$ such that $\bar{v}_{i}(y) \geq v_{i}(y)$ for all $y \in X$. 
We distinguish between payments and prices. A payment is a transfer from an agent to the seller. A vector of agent payments is an element of $\mathbf{R}^{n}$. Prices, on the other hand, are defined over the space of bundles, and may be personalized in our setting. We write $p_{i}\left(x_{i}\right)$ to denote the price of bundle $x_{i}$ to agent $i$. Prices are elements of $\mathbf{R}^{X n}$, but in certain cases it will suffice to consider linear prices, described by elements of $\mathbf{R}^{M}$. In this case the price of a bundle $x_{i}$ at linear prices $p$ is the usual dot product $p \cdot x_{i}=\sum_{j \in M} p(j) x_{i}(j)$, and this is independent of the identity of the agent.

We will consider two important environments, in which the agents' valuations are all drawn from one the classes below. Lehmann et al. [19] provide an excellent treatment of the computational complexity of the allocation problem with these classes and some of their generalizations.

Unit-Demand. A unit-demand valuation is described by a vector $w_{i} \in \mathbf{R}_{+}^{M}$. The value of bundle $x_{i}$ is

$$
v_{i}\left(x_{i}\right)=\max _{\left\{j: x_{i}(j)>0\right\}} w_{i}(j)
$$

In words, the agent only values the first unit it obtains, so it will select the first unit of the available commodity it values the most.

Substitutes. A substitutes valuation satisfies the following "substitutes" condition, introduced by Kelso and Crawford [15]. For any two linear price vectors $p^{\prime} \geq p$ and $x_{i} \in \arg \max _{y \in X} v_{i}(y)-p \cdot y$, there is an $x_{i}^{\prime} \in$ $\arg \max _{y \in X} v_{i}(y)-p^{\prime} \cdot y$ such that $x_{i}^{\prime}(j) \geq x_{i}(j)$ for all $j \in M$ such that $p^{\prime}(j)=p(j)$. In words, demand does not decrease for any commodities that do not see a price increase.

When there is just a single commodity $(m=1)$, concaveextensible valuations are exactly those valuations that exhibit non-increasing marginal values, and they satisfy the substitutes condition. They can therefore be identified by a vector $w_{i} \in \mathbf{R}_{+}^{l}$ where $w_{i 1} \geq w_{i 2} \geq \ldots \geq w_{i l}$, describing the marginal value of each unit. Unit-demand valuations are concave-extensible and satisfy the substitutes condition. The substitutes condition implies the existence of a Walrasian equilibrium when $l=1$ (recall that units are indivisible) [15]. With multiple units, we need the additional assumption of concave-extensibility to guarantee that a Walrasian equilibrium exists [9].

\section{RESOURCE ALLOCATION}

The objective is to implement a feasible, efficient allocation. An allocation is a vector of bundles $x=\left(x_{1}, \ldots, x_{n}\right)$. An allocation is feasible if $\sum_{i \in N} x_{i}(j) \leq l$ for all $j \in M$ (units may be discarded at no cost). Let $\Gamma$ denote the set of feasible allocations. An allocation $x$ is efficient if

$$
x \in \arg \max _{y \in \Gamma} \sum_{i \in N} v_{i}\left(y_{i}\right) .
$$

In our model an efficient allocation always exists (because the set of feasible allocations is finite), but it may not be unique. The efficient rule is the correspondence that maps valuation profiles to efficient allocations.

Note that two valuation vectors $v_{i}, v_{i}^{\prime}$ represent the same preferences if they differ by a constant. We can therefore assume that each valuation class $\mathcal{V}_{i}$ is normalized: there is a bundle $x_{i}$ (typically the empty bundle) such that $v_{i}\left(x_{i}\right)=0$ for all $v_{i} \in \mathcal{V}_{i}$. Translating a valuation by a constant does not change the set of efficient allocations according to (1), as expected.

\subsection{Implementation}

With selfish agents, it is not enough to simply query the agents for their private information and then implement an efficient allocation with respect to their reports. Ignoring for the moment the issue of communication - a problem with direct revelation - there is the issue that agents would overstate their values to gain a better outcome for themselves individually. A resource allocation scheme can be augmented with payments to incentivize the agents to report truthfully.

The Vickrey-Clarke-Groves (VCG) mechanism [5, 11, 30] is the following procedure. Each agent $i$ reports a valuation $\tilde{v}_{i} \in \mathcal{V}_{i}$. The seller implements an efficient allocation $x$ with respect to $\tilde{v}$ and charges agent $i$ the payment

$$
\hat{q}_{i}=\sum_{j \neq i} \tilde{v}_{j}\left(x_{j}^{-i}\right)-\sum_{j \neq i} \tilde{v}_{j}\left(x_{j}\right),
$$

where $x^{-i}$ is an efficient allocation of the items among agents $N-i .^{3}$ We call (2) agent $i$ 's VCG payment. More generally, a Groves scheme [11] charges the payment

$$
\hat{q}_{i}+h_{i}\left(\tilde{v}_{-i}\right)
$$

to agent $i$. Here $h_{i}$ is an arbitrary function that does not depend on agent $i$ 's report. The VCG mechanism is the Groves scheme with $h_{i} \equiv 0$. Note that (2) depends on the particular efficient allocation $x$ chosen, but not on the choice of efficient allocation $x^{-i}$ among agents $N-i$. We must therefore make reference to the associated allocation $x$ when talking of VCG payments. Note also that (2) is invariant to any renormalization of the agents' valuations - this is an added feature of the VCG mechanism that does not hold for Groves schemes in general.

It is well-known that truthful reporting is a dominant strategy equilibrium of any Groves scheme. Moreover, if the environment is smoothly path-connected, any efficient, strategyproof direct mechanism must be a Groves scheme [10, 13]. ${ }^{4}$ The VCG mechanism, in particular, has several attractive properties that make it central in the theory of mechanism design. In a single-item setting, it corresponds to Vickrey's second-price sealed-bid auction [30]. In our setting, the VCG mechanism is individually-rational and does not run a deficit. Among Groves schemes, the VCG mechanism is the unique mechanism that satisfies the natural condition that agents who acquire no items pay zero [3]. Finally, among all individually-rational Groves schemes, it also maximizes the revenue to the seller [16].

We have so far discussed direct mechanisms, where the available strategy of each player $i$ is to report a valuation from $\mathcal{V}_{i}$. To save on communication, the mechanism designer may turn to an indirect mechanism, where agents report messages from a space other than $\mathcal{V}$, and possibly several messages in sequence. The strategy of an agent in this case is a complete contingent plan of the messages the agent would send for each possible history of messages it could face. If a strategy profile leads to an efficient allocation with associated VCG payments (with respect to the

\footnotetext{
${ }^{3}$ The notation $N-i$ is shorthand for $N \backslash\{i\}$.

${ }^{4}$ All environments considered in this paper are smoothly path-connected.
} 
agents' true valuations), then this strategy profile becomes an ex post Nash equilibrium of the game induced by the mechanism [8]. So VCG payments are also relevant to indirect mechanisms. As mentioned in Section 1, for various environments, there are several auction designs that compute VCG payments together with the efficient allocation to bring sincere bidding into an equilibrium [1, 2, 7, 22].

Therefore, with incentives in mind, one outcome space we will consider is $\Gamma \times \mathbf{R}^{n}$, namely feasible allocations together with payments. The $V C G$ rule is the correspondence that maps valuations to efficient allocations with corresponding VCG payments.

\subsection{Realization}

We consider the "verification scenario". An omniscient oracle knows the private information of each agent, and can therefore identify a "desirable" outcome according to some pre-specified rule, such as the efficient or VCG rule. The oracle must prove to an outsider that his chosen outcome is indeed desirable. To do this he sends a message to the agents, which they will unanimously accept if and only if the designated outcome is desirable with respect to their private information. In this sense, the protocol verifies the chosen outcome for the outsider when all agents accept the message.

Let $\mathcal{O}$ denote the space of outcomes. A nondeterministic communication protocol $[17,24]$ is a triple $\Pi=\langle\mathcal{M}, \mu, h\rangle$. Here $\mathcal{M}$ is a message set, $\mu: \mathcal{V} \rightarrow \mathcal{M}$ is a message correspondence, and $h: \mathcal{M} \rightarrow \mathcal{O}$ is the outcome function. The message correspondence $\mu$ has the following properties:

- Existence: $\mu(v) \neq \emptyset$ for all $v \in \mathcal{V}$.

- Privacy preservation: $\mu(v)=\bigcap_{i} \mu_{i}\left(v_{i}\right)$ for all $v \in \mathcal{V}$, where $\mu_{i}: \mathcal{V} \rightarrow M$ for all $i \in N$.

Protocol $\Pi$ realizes choice rule $F: \mathcal{V} \rightarrow \mathcal{O}$ if $h(\mu(v)) \subseteq F(v)$ for all $v \in \mathcal{V}$. In a protocol that realizes choice rule $F$, the fact that the agents all accept a message $m$-where $i$ accepts $m$ if and only if $m \in \mu_{i}\left(v_{i}\right)$-verifies that outcome $h(m)$ is correctly chosen, because $h(m) \in h(\mu(v)) \subseteq F(v)$.

The notion of a nondeterministic communication protocol arises in both economics and computer science. The former literature uses continuous communication, measured as the amount of real numbers used to transmit a message. The latter uses discrete communication, measured in bits. We use continuous communication because it makes for clearer statements of our results.

The communication requirement of a protocol with continuous communication is the dimension of its message space. (Note that if a message contains an element drawn from a discrete set, this does not add to the communication burden by this definition.) To characterize this dimension, we need to provide a topology on the message space. Following NS, we use the topology induced by the following metric: the distance between messages $m$ and $m^{\prime}$ is

$$
\max \left\{\rho\left(\mu^{-1}(m), \mu^{-1}\left(m^{\prime}\right)\right), \rho\left(\mu^{-1}\left(m^{\prime}\right), \mu^{-1}(m)\right)\right\}
$$

where

$$
\rho\left(V, V^{\prime}\right)=\sup _{v \in V} \inf _{v^{\prime} \in V^{\prime}}\left\|v-v^{\prime}\right\|_{\infty}
$$

for $V, V^{\prime} \subseteq \mathcal{V}$. The motivation given by NS for this definition is that small changes in a message cannot drastically distort its "meaning": if message $m^{\prime}$ is close to $m$, there is a valuation consistent with $m^{\prime}$ that is close to a valuation consistent with $m$. This rules out the possibility of "dimension smuggling": using a lower-dimensional space to communicate high-dimensional vectors.

The communication requirement of a choice rule is the smallest communication requirement over all protocols realizing the rule. This will depend upon the environment $\mathcal{V}$. Clearly, the communication requirement of a given choice rule will be (weakly) larger for environment $\mathcal{V}^{\prime}$ than environment $\mathcal{V}$ if $\mathcal{V}^{\prime} \supseteq \mathcal{V}$. It should also be clear that the VCG rule requires at least as much communication as the efficient rule, because the VCG outcome specifies an efficient allocation. Additional communication may be required to verify VCG payments. Following Fadel and Segal [8], we call this added communication the overhead imposed by VCG payments.

\section{PRICE EQUILIBRIA}

We can now begin to characterize the communication requirement of the VCG rule. Our approach is modeled on that of NS for the efficient rule. They first draw a connection between the efficient rule and the competitive equilibrium rule. A competitive equilibrium $(\mathrm{CE})$ is an allocation-price pair $\langle x, p\rangle \in \Gamma \times \mathbf{R}^{X n}$ such that

$$
v_{i}\left(x_{i}\right)-p_{i}\left(x_{i}\right) \geq v_{i}\left(x_{i}^{\prime}\right)-p_{i}\left(x_{i}^{\prime}\right)
$$

for all $i \in N$ and all $x_{i}^{\prime} \in X$, and

$$
\sum_{i \in N} p_{i}\left(x_{i}\right) \geq \sum_{i \in N} p_{i}\left(x_{i}^{\prime}\right)
$$

for all $x^{\prime} \in \Gamma$.

In words, (3) says that bundle $x_{i}$ maximize agent $i$ 's utility at prices $p$, and (4) says that $x$ maximizes the seller's revenue at prices $p$. A Walrasian equilibrium is a CE such that prices $p$ are linear. If $\langle x, p\rangle$ is a $\mathrm{CE}$, we say that prices $p$ "support" allocation $x$. In our model, there always exists a CE with nonlinear personalized prices [4]. The Fundamental Welfare Theorems say that: (1) if prices $p$ support an allocation $x$, the allocation is efficient, and (2) there always exist supporting prices $p$ for an efficient allocation $x$ [21]. Bikhchandani and Ostroy [4] give proofs of these theorems for our model. Furthermore, it is not hard to show that if prices $p$ support some efficient allocation, then they support all efficient allocations. Thus we can speak of CE prices $p$ without reference to any allocation, in contrast to VCG payments. Note also that renormalizing CE prices (translating any agent $i$ 's personalized prices $p_{i}$ by a constant) again gives $\mathrm{CE}$ prices. Like valuations, we can therefore assume that $\mathrm{CE}$ prices are normalized.

The $C E$ rule maps valuations to the associated set of competitive equilibria. The outcome space here is $\Gamma \times \mathbf{R}^{X n}$. A nondeterministic communication protocol for this rule is immediate from the definition of a CE. Suppose the oracle wishes to prove to the outsider that $\langle x, p\rangle$ is a CE. It can use the following "basic CE protocol":

- The oracle broadcasts $\langle x, p\rangle$ as his message.

- Each agent $i$ accepts the message if and only if $x_{i}$ maximizes its utility at prices $p$, and $x$ maximizes revenue at prices $p$ (any agent can see the latter for himself).

The communication requirement of this protocol is the dimension of the transmitted prices $p$-recall that transmit- 
ting discrete allocations adds nothing to the communication burden. NS showed the following.

THEOREM 1. [24] The communication requirements of the efficient and CE rules are identical.

The actual statement of their result says that given a protocol for the efficient rule, there is a protocol for the CE rule with the same message space, and vice-versa. ${ }^{5}$ This result is very useful for characterizing the communication requirement of the efficient rule for various environments, because it is often easier to instead characterize the communication requirement of the $\mathrm{CE}$ rule.

For the VCG rule, the relevant price equilibrium concept is universal competitive equilibrium (UCE), introduced by Parkes and Ungar [26]. A UCE is a CE $\langle x, p\rangle$ such that prices $p$ also support any efficient allocation $x^{-i}$ of the units among agents $N-i$. In the sequel, we will refer to the economy with agents $N$ as the "main economy", and the economies with agents $N-i$ for each $i \in N$ as the "marginal economies".

Again, a nondeterministic communication protocol verifying the UCE rule is immediate. Let $x$ be efficient for the main economy, and let $x^{-i}$ be efficient for the marginal economy with agents $N-i$, for all $i \in N$. Let $p$ be UCE prices. To prove that $\langle x, p\rangle$ is a UCE, we can use the following "basic UCE protocol":

- The oracle broadcasts $\left\langle x, x^{-1}, \ldots, x^{-n}, p\right\rangle$.

- Each agent $i$ accepts the message if and only if $x_{i}$ and $x_{i}^{-k}$ maximize its utility at prices $p$, for each $k \neq i$, and allocations $x, x^{-k}$ for $k \in N$ all maximize revenue at prices $p$.

In analogy to Theorem 1, we have the following.

THEOREM 2. The communication requirements of the VCG and UCE rules are identical.

This follows from two statements that have already appeared in the literature on UCE prices. Mishra and Parkes [22] showed the following.

THEOREM 3. [22] Let $\langle x, p\rangle$ be a universal competitive equilibrium, and let $x^{-i}$ be any revenue-maximizing allocation of the items among agents $N-i$ at prices $p$. Then agent $i$ 's $V C G$ payment is

$$
\hat{q}_{i}=\sum_{j \in N-i} p_{j}\left(x_{j}^{-i}\right)-\sum_{j \in N-i} p_{j}\left(x_{j}\right) .
$$

Note that the first term in (5) does not depend on the revenue-maximizing allocation $x^{-i}$ chosen, so it can be computed with knowledge of UCE prices $p$ only. Therefore, with knowledge of UCE prices, the outsider can reconstruct the agents' VCG payments to verify the outcome designated by the oracle. Lahaie et al. [18], meanwhile, proved the following.

THEOREM 4. [18] If communication protocol $\langle\mathcal{M}, \mu,\langle f, q\rangle\rangle$ realizes the VCG rule, where $f$ is the allocation function and $q$ is the payment function, then there is an assignment $p: \mathcal{M} \rightarrow \mathbf{R}^{X n}$ of prices to messages such that protocol $\langle\mathcal{M}, \mu,\langle f, p\rangle\rangle$ realizes the UCE rule.

${ }^{5}$ Parkes [25] independently established the same result for a slightly more restricted model.
This last result shows that the communication requirement of the UCE rule is no greater than that of the VCG rule, whereas Theorem 3 implies the reverse. This proves Theorem 2.

\section{NAÏVE PROTOCOLS}

Before turning to specific environments, we give a general bound on the overhead of VCG payments. Suppose the oracle wishes to prove that $x$ is efficient and that the corresponding VCG payments are $\hat{q}$ as defined by (2). Let $x^{-1}, \ldots, x^{-n}$ be efficient allocations for each of the marginal economies. Suppose also that the oracle has a protocol $\Pi$ for the efficient rule. The following "naïve" protocol (not to be confused with the "basic protocols" of the previous section) verifies VCG outcome $\langle x, \hat{q}\rangle$.

- The oracle sends messages $m, m^{-1}, \ldots, m^{-n}$ that verify that $x, x^{-1}, \ldots, x^{-n}$ are efficient for their corresponding economies, respectively, using $\Pi$.

- The oracle sends $v_{i}^{\prime}\left(x_{i}^{-k}\right)$ for each $i \in N$ and $k \neq i$, where $v_{i}^{\prime}\left(y_{i}\right)=v_{i}\left(y_{i}\right)-v_{i}\left(x_{i}\right)$ for each $y_{i} \in X$.

- Each agent $i$ accepts the complete message if an only if

1. It accepts each $m, m^{-1}, \ldots, m^{-n}$ according to $\Pi$.

2. It is indeed the case that the values sent satisfy $v_{i}^{\prime}\left(x_{i}^{-k}\right)=v_{i}\left(x_{i}^{-k}\right)-v_{i}\left(x_{i}\right)$ for $k \neq i$.

At most $m l$ agents get a nonempty bundle in $x$, and removing an agent that receives nothing in $x$ does not change the efficient allocation. Therefore the leading messages can be transmitted with $(\min \{n, m l\}+1) d$ real numbers, where $d$ is the communication requirement of the efficient rule. The number of trailing values transmitted is $\min \left\{n(n-1), m^{2} l^{2}\right\}{ }^{6}$ This gives us the following result.

Proposition 1. The communication requirement of the $V C G$ rule (equivalently, the UCE rule) is at most

$$
(\min \{n, m l\}+1) d+\min \left\{n(n-1), m^{2} l^{2}\right\},
$$

where $d$ is the communication requirement of the efficient rule (equivalently, the CE rule).

There is a parallel naïve protocol for the UCE rule, based on a construction of UCE prices from CE prices for the main and marginal economies. Consider Figure 1, which shows a valuation for a single commodity exhibiting decreasing marginal values. Geometrically, condition (3) means that CE prices define a supporting hyperplane at the agent's allocated bundle. In this case prices of dimension 1 suffice to support any single bundle. Now suppose that the agent gets one item in the main economy, and three items in some marginal economy. UCE prices must support each of these bundles, but clearly no prices of dimension 1 can do this.

\footnotetext{
${ }^{6}$ We conjecture that for substitutes valuations - which covers all valuations considered in this paper - this can be improved to $\min \{n(n-1), m l\}$. This can be shown for the environment where $m=1$ and the agents' valuations exhibit decreasing marginal values, as in Section 6.1.2, as follows. Since $x_{i}^{-k} \geq x_{i}$ for each $i \neq k$ by the arguments of Section $6.1 .2, x^{-k}$ can differ from $x$ by only at most $x_{k}$ bundles. Therefore the number of additional values that must be communicated is at most $\sum_{k \in N} x_{k} \leq l$.
} 


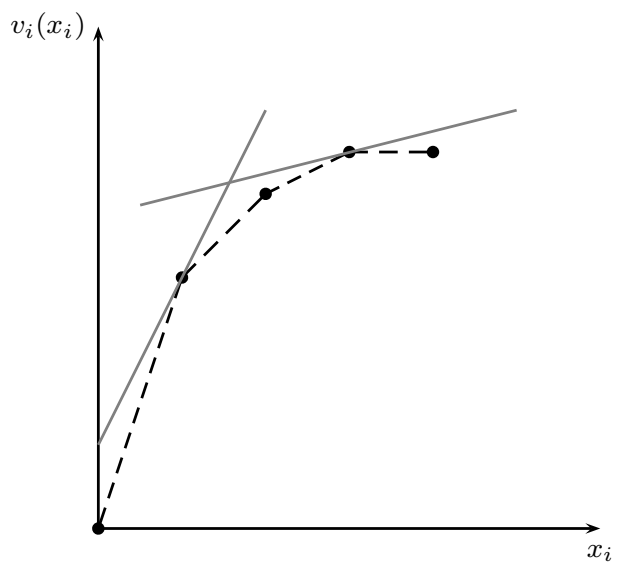

Figure 1: To construct UCE prices from CE prices for the main and marginal economies, we take the lower envelope of the latter. The resulting prices support all the necessary allocations (here, 1 unit and 3 units).

The figure suggests, however, that taking the lower envelope of the $\mathrm{CE}$ prices for the main and marginal economies could yield $\mathrm{CE}$ prices. In the following result, $k=0$ refers to the main economy.

LEMMA 1. Let $\left\langle x^{-k}, p^{-k}\right\rangle$ be a competitive equilibrium for economy $k$, for $k=0, \ldots, n$. Let $\pi_{i}^{-k}=v_{i}\left(x_{i}^{-k}\right)-p_{i}^{-k}\left(x_{i}^{-k}\right)$ be the payoff of agent $i \neq k$ in economy $k$. Then the following are UCE prices:

$$
p_{i}(y)=\min _{k \neq i}\left\{p_{i}^{-k}(y)+\pi_{i}^{-k}\right\} .
$$

Proof. We first claim that $p_{i}\left(x_{i}^{-k}\right)=p_{i}^{-k}\left(x_{i}^{-k}\right)+\pi_{i}^{-k}$ for $k=0, \ldots, n$. If this were not the case there would be an $s \neq k$ such that

$$
\begin{aligned}
& p_{i}^{-s}\left(x_{i}^{-k}\right)+\pi_{i}^{-s}<p_{i}^{-k}\left(x_{i}^{-k}\right)+\pi_{i}^{-k} \\
\Rightarrow & \pi_{i}^{-s}<v_{i}\left(x_{i}^{-k}\right)-p_{i}^{-s}\left(x_{i}^{-k}\right)
\end{aligned}
$$

which is a contradiction, because $\pi_{i}^{-s}$ is the maximum utility that $i$ can attain at prices $p_{i}^{-s}$. We thus find that

$$
\begin{aligned}
v_{i}\left(x_{i}^{-k}\right)-p_{i}\left(x_{i}^{-k}\right) & =v_{i}\left(x_{i}^{-k}\right)-p_{i}^{-k}\left(x_{i}^{-k}\right)-\pi_{i}^{-k} \\
& =0
\end{aligned}
$$

for $k=0, \ldots, n$ and $i \neq k$. Now let $x_{i}$ be an arbitrary bundle, and let $s$ be the index at which the minimum is reached for $x_{i}$ on the right-hand side of (7). We have

$$
\begin{aligned}
v_{i}\left(x_{i}\right)-p_{i}\left(x_{i}\right) & =v_{i}\left(x_{i}\right)-p_{i}^{-s}\left(x_{i}\right)-\pi_{i}^{-s} \\
& \leq v_{i}\left(x_{i}^{-s}\right)-p_{i}^{-s}\left(x_{i}^{-s}\right)-\pi_{i}^{-s} \\
& =0 \\
& =v_{i}\left(x_{i}^{-k}\right)-p_{i}\left(x_{i}^{-k}\right)
\end{aligned}
$$

for $k=0, \ldots, n$, where the inequality follows from the fact that $x_{i}^{-s}$ maximizes agent $i$ 's utility at prices $p_{i}^{-s}$. Since $x_{i}$ was arbitrary, $x_{i}^{-k}$ maximizes agent $i$ 's utility at prices $p_{i}$, for $k=0, \ldots, n$.

Finally, fix $k \in\{0, \ldots, n\}$ and let $x$ be an arbitrary feasible allocation among agents $N-k$. We have

$$
\begin{aligned}
\sum_{i \in N-k} p_{i}\left(x_{i}\right) & \leq \sum_{i \in N-k} p_{i}^{-k}\left(x_{i}\right)+\sum_{i \in N-k} \pi_{i}^{-k} \\
& \leq \sum_{i \in N-k} p_{i}^{-k}\left(x_{i}^{-k}\right)+\sum_{i \in N-k} \pi_{i}^{-k} \\
& =\sum_{i \in N-k} p_{i}\left(x_{i}^{-k}\right)
\end{aligned}
$$

where the first inequality follows from the definition of $p$ and the second from the fact that $x^{-k}$ maximizes revenue at prices $p^{-k}$. Hence $x^{-k}$ maximizes revenue among allocations to agents $N-k$ at prices $p$, for $k=0, \ldots, n$. This shows that $p$ are $\mathrm{UCE}$ prices.

If $\mathrm{CE}$ prices are of dimension $d$, prices (7) are of dimension (6), mirroring the naïve VCG protocol. One would hope that cleverer protocols exist that perform better than the naïve protocols. In the next section, we will see that this hope is not justified for some environments: decreasing marginal values, and substitutes valuations. The naïve protocols' communication requirement is asymptotically optimal for these environments for certain parameter settings of $l, m$, and $n$.

\section{APPLICATIONS}

We consider settings with homogeneous commodities $(m=$ 1) and heterogeneous commodities in turn, and for each of these the cases with single and multiple units, because their associated environments exhibit different communication requirements. Single-unit settings impose no overhead, while multi-unit settings have positive overhead.

To prove lower bounds, we use a "fooling set" technique common in the computer science literature [17], and inspired by NS. A fooling set $\mathcal{V}^{*} \subseteq \mathcal{V}$ has the property that for any two $v, v^{\prime} \in \mathcal{V}^{*}$, we have $\mu(v) \cap \mu\left(v^{\prime}\right)=\emptyset$. This means that the restriction of $\mu$ to $\mathcal{V}^{*}$ has an injective selection $\sigma: \mathcal{V}^{*} \rightarrow$ $\mu\left(\mathcal{V}^{*}\right)$. If $\mathcal{V}$ is normalized and $\sigma^{-1}$ is Lipschitz continuous, we then have $\operatorname{dim} \mathcal{M} \geq \operatorname{dim} \mu\left(\mathcal{V}^{*}\right) \geq \operatorname{dim} \mathcal{V}^{*}$, as NS explain (see their Proposition 2). The challenge is in constructing the fooling set. The injection $\sigma$ will be obvious from our 
constructions, as well as the fact that its inverse is Lipschitz continuous. We will not find need to make $\sigma$ explicit.

\subsection{Homogeneous Goods}

\subsubsection{Single-Unit}

We first consider the simple case with $l=1$ units. This is the problem of allocating a single item. This case is essentially trivial, but serves to nicely illustrate our techniques in the simplest possible setting. We first consider an artificial, restricted environment to show how overhead can be incurred, then note that in the usual environment there is no overhead.

Consider the environment with two agents where agent 1 always has a value of 1 for the item, while agent 2's value is $w_{2} \in[0,1)$. It is always efficient to allocate the item to agent 1 , so no communication is needed to verify this efficient outcome. If agent 1 is removed, agent 2 gets the item. UCE Prices $p$ must therefore support both one unit and zero units for agent 2: $w_{2}-p_{2}(1)=0-p_{2}(0)$, or $p_{2}(1)=w_{2}$ after normalization. The dimension of the UCE price space is therefore at least the dimension of agent 2's valuation space, which is 1 . Setting a price of $w_{2}$ on the item gives a UCE price, so the dimension of UCE prices is at most 1, matching the lower bound. By Theorem 2, the overhead of VCG payments is 1 real number in this example.

The technique used in this example is typical. We construct an environment where an agent obtains one more unit in a marginal economy than in the main economy. Because UCE prices must support both these allocations, the marginal price of the unit must equal the agent's marginal value for the unit. Therefore different valuation profiles must lead to different UCE outcomes, and we have a fooling set. See Figure 2 for a geometric interpretation.

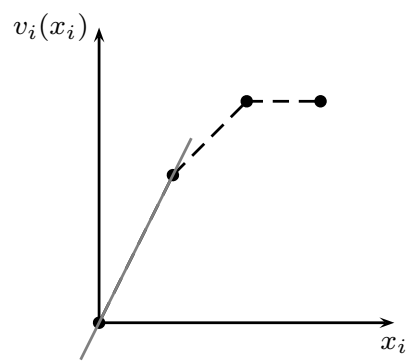

Figure 2: If UCE prices support both 0 units and 1 unit, the slope of the prices must equal the marginal value of the first unit.

In the usual environment where agent values are drawn from $[0,1]$, the communication requirements of the efficient and VCG rules (equivalently, of the CE and UCE rules) are both 1 real number, so there is no overhead. The secondhighest value for the item is always a UCE price.

\subsubsection{Multi-Unit}

When $l>1$, however, there is a positive overhead. Assume that agent valuations exhibit decreasing marginal values. It is well-known that $\mathrm{CE}$ prices of dimension 1 exist in this case, even if units are indivisible. A nice way to see this is via the greedy algorithm, which correctly constructs an efficient allocation. Each unit is allocated in sequence to the agent whose marginal value for the unit is greatest (breaking ties arbitrarily). Let $x$ be the efficient allocation that results from this procedure. Assume (w.l.o.g.) that agent 1 received the last unit, and that agent 2 would have received the next unit if there were any more. Then any $p \in\left[v_{2}\left(x_{2}+1\right)-v_{2}\left(x_{2}\right), v_{1}\left(x_{1}\right)-v_{1}\left(x_{1}-1\right)\right]$ is a CE price, giving us an upper bound of 1 (the lower bound of 1 is immediate).

Combined with Theorem 2, the following lemma gives an upper bound on communication for the VCG rule.

LEMma 2. With decreasing marginal values, there exist UCE prices of dimension $l$.

Proof. As explained above, the greedy algorithm produces an efficient allocation $x$. Assume (w.l.o.g.) that agent 1 would receive the next unit if there were any, and let $a=v_{1}\left(x_{1}+1\right)-v_{1}\left(x_{1}\right)$. The UCE prices $p$ we will construct are personalized. Each $p_{i}$ depends only on the number of units and exhibits decreasing marginal prices, like the agents' valuations.

The price construction is as follows. The marginal price of the first $x_{i}$ units to agent $i$ is $a$. Let $x^{-1}, \ldots, x^{-n}$ be efficient allocations for the marginal economies. Allocating items greedily in marginal economy $k$ in the same sequence as for the main economy (and breaking ties the same way), we see that we can assume $x_{i}^{-k} \geq x_{i}$ for $k \neq i$. For each $i$, let $y_{i}=\max _{k \neq i} x_{i}^{-k}$. The marginal prices of units $x_{i}+1$ to $y_{i}$ to agent $i$ are the marginal values of the units the agent. Note that at least one of these marginal prices is $a$. Finally, the marginal price of any unit beyond $y_{i}$ for each agent $i$ is $\max _{k}\left\{v_{k}\left(y_{k}\right)-v_{k}\left(y_{k}-1\right)\right\}$. This completes the price construction.

The construction ensures that each agent is indifferent between obtaining anything from $x_{i}$ to $y_{i}$ units, because the marginal surplus of each of these units is zero. It also ensures that anything in this range is (weakly) preferred to less than $x_{i}$ units or more than $y_{i}$ units. Thus the allocated bundles maximize the agents' utilities in the main and marginal economies. To compute a revenue-maximizing allocation, we can again use the greedy algorithm. The prices are constructed such that the sequence of assignments is identical to the sequence of assignments used to determine the efficient outcome, for the main and marginal economies, so the efficient allocations are also revenue-maximizing. The constructed prices are therefore UCE prices.

To specify the prices, we need to communicate $y_{i}-x_{i}$ real numbers for each agent $i \in N$. We have

$$
\begin{aligned}
\sum_{i}\left(y_{i}-x_{i}\right) & \leq \sum_{i} \sum_{k \neq i}\left(x_{i}^{-k}-x_{i}\right) \\
& =\sum_{k} \sum_{i \neq k}\left(x_{i}^{-k}-x_{i}\right) \\
& =\sum_{k} x_{k} \\
& =l
\end{aligned}
$$

The last two equalities assume (w.l.o.g.) that all items are allocated. This completes the proof.

To get a lower bound, consider now the environment where agent 1 always has a marginal value of 1 for each unit, and agents $i=2, \ldots, n$ have unit demand. The marginal value of agent $i \neq 1$ for its first unit is $w_{i} \in[0,1)$. It is efficient to give all units to agent 1 . Let $r=\min \{l, n-1\}$. If agent 
1 is removed, agents $2, \ldots, r+1$ each get one unit. So for $i=2, \ldots, r+1$, UCE prices $p$ must satisfy

$$
\begin{aligned}
v_{i}(1)-p_{i}(1) & =v_{i}(0)-p_{i}(0) \\
\Rightarrow \quad p_{i}(1)-p_{i}(0) & =w_{i}
\end{aligned}
$$

Normalizing prices so that $p_{i}(0)=0$ for each $i \in N$, we see that the dimension of the UCE price space must be at least the dimension of the space of valuations for agents $2, \ldots, r+1$, namely $r$.

The agents' valuations in this environment exhibit decreasing marginal values, so the lower bound holds for this valuation class in general.

Proposition 2. The communication requirements of the $V C G$ rule with a single commodity and decreasing marginal values is at least $\min \{l, n-1\}$.

As we saw above, the communication requirement of the efficient rule is 1 in this environment, so computing VCG payments imposes an $r$-fold increase in communication, in the worst case. This overhead is positive for $l>1$. The lower bound matches the upper bound of Lemma 2 for $n>l$, and the communication requirement is exactly $l$. In this case, the naïve protocol gives an upper bound of $2 l+1$ according to Proposition 1 (see also footnote 6), so the naïve protocol is asymptotically optimal. An exact characterization for the case of $n \leq l$ remains an open question. We conjecture that the lower bound is tight.

\subsection{Heterogeneous Goods}

\subsubsection{Single Unit}

The single-unit setting with heterogeneous goods is the assignment problem, and the relevant environment is unit demand. It is well known that a Walrasian equilibrium exists in this environment. There are two striking facts about Walrasian prices in the assignment problem. First, the set of Walrasian prices forms a lattice [29]. In particular, there are minimum and maximum Walrasian prices. Second, minimum Walrasian prices $\underline{p} \in \mathbf{R}_{+}^{M}$ coincide with VCG payments: if agent $i$ obtains item $j$ in the efficient assignment, then the VCG payment of agent $i$ is $p(j)$ [20].

First we characterize the communication requirement of efficiency (equivalently, of the CE rule). For $n>m$, the following is well known [29], but we have not come across the characterization for $n \leq m$.

Lemma 3. The minimum Walrasian prices have dimension $\min \{m, n-1\}$ in the assignment problem.

Proof. Since the case $n>m$ is known, assume $n \leq m$. Consider the minimum Walrasian prices $\underline{p}$, and assume items $S \subseteq M$ are allocated, where $|S|=n$. The $m-n$ unallocated items must have a shadow price of 0 , by standard arguments from linear programming. We cannot have $p(j)>0$ for all $j \in S$. Otherwise $\underline{p}(j)-\epsilon \sum_{j \in S} e_{j}$ would also be Walrasian prices for some $\epsilon>0$, and this would contradict the minimality of $p$. So there is a $j \in S$ with $p(j)=0$, and $n-1$ dimensions suffice.

A matching lower bound is obtained with the following fooling set. All agents have the same unit-demand valuation corresponding to $w \in[0,1]^{S} \times\{0\}^{M \backslash S}$, where $S$ is a subset of items of size $r=\min \{m, n-1\}$. Note that any assignment of the items to the agents (where each agent gets exactly one item, and any remaining items are discarded) is efficient. Therefore, by Proposition 2 of NS [24], the communication requirement of the efficient rule is at least the dimension of the fooling set, namely $r$.

Proposition 3. The communication requirement of the efficient rule in the assignment problem is $\min \{m, n-1\}$.

We now turn to the communication requirement of the VCG rule, or equivalently, the UCE rule. Since minimum Walrasian prices $p$ coincide with VCG payments, it may seem that simply transmitting $p$ in the basic CE protocol of Section 4 would suffice to verify the VCG outcome, giving us an upper bound via Lemma 3. But this is not a valid protocol: it does not prove to the outsider that the declared prices $p$ are in fact minimal, just that they are Walrasian. To derive a protocol that also proves minimality, we appeal to the following result. In the proof and what follows, $e_{j}$ is the unit vector with a 1 in component $j$ and 0 's in all others.

Lemma 4. Minimum Walrasian prices are UCE prices in the assignment problem.

Proof. Walrasian prices $\underline{p}$ support the efficient assignment $\sigma: M \rightarrow N$ with all agents present, by definition. We will show that $\underline{p}$ also support an efficient assignment of items $M$ to agents $N-1$. For each item $j$, let $d(j)=\{i \in$ $\left.N-1: j \in D_{i}(p)\right\}$, where $D_{i}(p)$ is the demand set of agent $i$ at prices $p$. Assume for the moment that for all $T \subseteq M$,

$$
\left|\bigcup_{j \in T} d(j)\right| \geq|T| .
$$

It then follows from Hall's theorem that for each $j \in M$, we can select a distinct $i \in N-1$ such that $j \in D_{i}(p)$. Prices $\underline{p}$ therefore support this assignment of items to agents $N-1$, and by the same argument any efficient assignment to agents $N-i$, for all $i \in N$, so $\underline{p} \mathrm{UCE}$ are prices.

It remains to be shown that (8) holds. Assume (w.l.o.g.) that $\sigma(1)=1$. For $T \subseteq M-1$, we have $\left|\bigcup_{j \in T} d(j)\right| \geq$ $\left|\bigcup_{j \in T}\{\sigma(j)\}\right| \geq|T|$, because $\underline{p}$ supports $\sigma$ and the latter is one-to-one. So let $T \ni 1$. We claim that

$$
\bigcup_{j \in T} d(j) \backslash \bigcup_{j \in T}\{\sigma(j)\} \neq \emptyset .
$$

(Note that this then implies (8).) Assume for the sake of contradiction that (9) does not hold. Then there is a sufficiently small $\epsilon>0$ such that for all $i \in N \backslash \bigcup_{j \in T} d(j)$, all $k \in D_{i}(p)$, and all $j \in T$, we have

$$
v_{i}\left(e_{k}\right)-\underline{p} \cdot e_{k}>v_{i}\left(e_{j}\right)-\underline{p} \cdot e_{j}+\epsilon .
$$

Therefore if we let $p^{\prime}=\underline{p}-\epsilon \sum_{j \in T} e_{j}, D_{i}\left(p^{\prime}\right)=D_{i}(p)$ for $i \in$ $N \backslash \bigcup_{j \in T}$. For each agent $i \in \bigcup_{j \in T} d(j), k=\sigma^{-1}(i)$ remains in its demand set at prices $p^{\prime}$ because $k$ sees a price decrease. Therefore $p^{\prime}$ supports $\sigma$, contradicting the minimality of $\underline{p}$. This completes the proof.

Lemma 4 could also be proved by contradiction, using Leonard's [20] result that minimum Walrasian prices equal VCG payments in the assignment problem. We prefer the above proof because it is more elementary, making direct appeal to the definition of a UCE. It implies Leonard's result 
as a corollary: since minimum Walrasian prices $p$ are $\mathrm{UCE}$ prices, by Theorem 3 we then have

$$
p_{j}=\sum_{k} p_{k}-\sum_{k \neq j} p_{k}=\hat{q}_{i}
$$

where $i$ is the agent assigned item $j$. Hence UCE prices are unique, and the price of an item is the VCG payment of the agent that receives it.

For our purposes, the import of Lemma 4 is that the UCE rule can be verified with a message space of dimension $r$ via the basic UCE protocol of Section 4, because there always exists a minimum Walrasian price vector. This gives an upper bound of $r$ for the VCG rule, by Theorem 2. The lower bound for the $\mathrm{CE}$ price rule also applies to the UCE rule, since a UCE is a CE, and this gives us an exact characterization.

Proposition 4. The communication requirement of the $V C G$ rule in the assignment problem with unit demand is $\min \{m, n-1\}$.

Comparing with Proposition 3, we see that VCG payments impose no overhead in the assignment problem.

\subsubsection{Multi-Unit}

The relevant environment here is concave-extensible, substitutes valuations. It is known that Walrasian prices of dimension $m$ exist in this case [9]. NS provide a matching lower bound of $m$ using a fooling set based on additive valuations, which are a subset of the substitutes valuations.

For the VCG rule, we first provide a lower bound. We assume $n>m$ for clarity; a simple modification gives the bound for $n \leq m$. Consider the following fooling set. We number the commodities $1, \ldots, m$. Each agent $i$ 's valuation is identified with a vector $w_{i} \in[0,1)^{M}$. The last agent has an additive valuation: $v_{n}\left(x_{n}\right)=\sum_{\left\{j: x_{n}(j)>0\right\}} w_{n}(j)$. Note that only the first unit of any commodity may have non-zero marginal value with this valuation. Each agent $i$ among the first $m$ agents has unit demand for each commodity $j \neq i$, and the marginal value of the first unit of $j$ is $w_{i}(j)$. The agent demands all $l$ units of commodity $i$ : the first $l-1$ have a marginal value of 1 , and the last has a marginal value of $w_{i}(i)$. The remaining agents $i=m+1, \ldots, n-1$ (if any), have unit demand valuations as specified by $w_{i}$. In addition, the valuation profile satisfies

$$
w_{i}(i)=w_{n}(i)>\max _{j \neq i, n} w_{j}(i)
$$

for $i=1, \ldots, m$. It is efficient to allocate one unit of each commodity to agent $n$, and the $l-1$ remaining units of commodity $i$ to agent $i$. Since giving agent $n$ 's unit of $i$ to agent $i$ also gives an efficient allocation, CE prices must support both of these allocations. In particular, we have

$$
w_{n}(i)-p_{n}(i)=0 \text {. }
$$

If we remove an agent $i$ among the first $m$ agents, $\min \{l-$ $1, n-2\}$ of the remaining agents besides $n$ each get one unit of $i$. For such agents $j \neq i, n$, UCE prices therefore satisfy

$$
\begin{aligned}
& w_{j}(i)+(l-1)-p_{j}\left(e_{i}+(l-1) e_{j}\right) \\
& =(l-1)-p_{j}\left((l-1) e_{j}\right) \\
\Rightarrow \quad & w_{j}(i)=p_{j}\left(e_{i}+(l-1) e_{j}\right)-p_{j}\left((l-1) e_{j}\right),
\end{aligned}
$$

if $j \leq m$, and otherwise

$$
w_{j}(i)-p_{j}\left(e_{i}\right)=0
$$

for $m<j \leq n-1$ (if any). Equation (10) pins down $m$ dimensions, while (11) and (12) together pin down an additional $m \min \{l-1, n-2\}$ dimensions. If we had $n \leq m$, a slight modification would give $(n-1) \min \{l-1, n-2\}$ for the latter, which yields the following result.

Proposition 5. The communication requirement of the VCG rule with substitutes valuations is at least

$$
\min \{m, n-1\} \min \{l-1, n-2\}+m .
$$

For $n>m$ and $l \geq n-1$, the lower bound is $(n-1) m$, representing an $(n-1)$-fold increase in communication over the efficient rule. We do not have a way to construct UCE prices for this environment besides the naïve construction given in Lemma 1. This is not necessarily a poor approach here. If $l=m=n$, the upper bound of the naïve protocol together with the lower bound of Proposition 5 show that the communication requirement of the VCG rule is $\Theta\left(n^{2}\right)$. For the efficient rule, we saw that communication is $\Theta(n)$ when $m=n$. Hence VCG payments can increase communication by an order of magnitude.

\section{CONCLUSION}

We have examined the nondeterministic communication requirements of realizing the VCG outcome in settings with indivisible units of different commodities. We leveraged the known equivalence between the communication requirements of the VCG and UCE rules, in analogy to the NS approach for the efficient rule. We gave a construction of UCE prices from CE prices from the main and marginal economies, yielding a naïve UCE protocol that mirrors the naïve VCG protocol, and a general bound on the overhead imposed by VCG payments.

We considered four different environments with concaveextensible valuations. In single-unit settings - the singleitem auction, and the assignment problem with unit-demand valuations - we found that VCG payments impose no overhead. This is a striking observation, and it would be of interest to understand if this is a general property of single-unit settings. It is also natural to consider substitutes valuations with just a single unit of each commodity, as in Kelso and Crawford's original model of firms and workers [15]. Perhaps this environment has a positive overhead; note though that Proposition 5 does not give any overhead when $l=1$. Mishra and Parkes [22] have shown that linear UCE prices do not exist in this case, but perhaps another, less conventional, low-dimensional subspace could encode UCE prices.

For multi-unit environments the characterizations are less precise. Matching upper and lower bound are still lacking for the environment with decreasing marginal values when $n \leq$ $l$. For $n>l$ our characterization is exact, and it shows that the naïve VCG protocol is asymptotically optimal. For the environment of substitutes valuations, we do not even know how to construct tailored UCE prices; only the construction of Lemma 1 is known. This is the most significant remaining open question. Nevertheless, if the number of units and commodities is on the order of the number of agents, we still find that the communication requirement of the VCG rule is quadratic in the number of agents, which is an order of magnitude increase over the efficient rule.

There are two broad avenues for future work. The first is to try to derive upper and lower bounds for deterministic communication protocols. Computing rather than verifying 
the VCG outcome should require a strict increase in communication, but we do not have any way to quantify this increase. The second would be to examine Groves schemes other than the VCG mechanism, with sufficient restrictions (e.g., individual-rationality, no-deficit) to disallow protocols such as Reichelstein's [27] mentioned in Section 1. It could be productive to characterize those Groves schemes that have an associated price equilibrium concept, just as there is UCE for the VCG mechanism, to understand the scope of the approach used in this paper.

\section{REFERENCES}

[1] Lawrence M. Ausubel. An efficient ascending-bid auction for multiple objects. American Economic Review, 94(5):1452-1475, 2004.

[2] Lawrence M. Ausubel. An efficient dynamic auction for heterogeneous commodities. American Economic Review, 96(3):602-629, 2006.

[3] Lawrence M Ausubel and Paul R Milgrom. Ascending auctions with package bidding. Frontiers of Theoretical Economics, 1:1-42, 2002.

[4] Sushil Bikhchandani and Joseph M. Ostroy. The package assignment model. Journal of Economic Theory, 107:377-406, 2002.

[5] E. H. Clarke. Multipart pricing of public goods. Public Choice, 11:17-33, 1971.

[6] Sven de Vries, James Schummer, and Rakesh V. Vohra. On ascending Vickrey auctions for heterogeneous objects. Journal of Economic Theory, 132(1):95-118, 2007.

[7] Gabrielle Demange, David Gale, and Marilda Sotomayor. Multi-item auctions. Journal of Political Economy, 94(4):863-872, 1986.

[8] Ronald Fadel and Ilya Segal. The communication cost of selfishness. Journal of Economic Theory, 2008. Forthcoming.

[9] Satoru Fujishige and Zaifu Yang. A note on Kelso and Crawford's gross substitutes condition. Mathematics of Operations Research, 28(3):463-469, 2003.

[10] Jerry Green and Jean-Jacques Laffont. Characterization of satisfactory mechansims for the revelation of preferences for public goods. Econometrica, 45:427-438, 1977.

[11] Theodore Groves. Efficient collective choice when compensation is possible. Review of Economic Studies, 46:227-241, 1979.

[12] Faruk Gul and Ennio Stacchetti. Walrasian equilibrium with gross substitutes. Journal of Economic Theory, 87:95-124, 1999.

[13] Bengt Holmstrom. Groves schemes on restricted domains. Econometrica, 47(5):1137-1144, 1979.

[14] Leonid Hurwicz. On the dimensional requirements of informationally decentralized Pareto-satisfatory processes. In K. J. Arrow and L. Hurwicz, editors, Studies in Resource Allocation Processes, pages 413-424. Cambridge University Press, 1977.
[15] Alexander S. Kelso and Vincent P. Crawford. Job matching, coalition formation, and gross substitutes. Econometrica, 50:1483-1504, 1982.

[16] Vijay Krishna and Motty Perry. Efficient mechanism design. Technical report, Pennsylvania State University, 2000.

[17] Eyal Kushilevitz and Noam Nisan. Communication Complexity. Cambridge University Press, 1997.

[18] Sébastien Lahaie, Florin Constantin, and David C. Parkes. More on the power of demand queries in combinatorial auctions: Learning atomic languages and handling incentives. In Proceedings of the 19th International Joint Conference on Artificial Intelligence, Edinburgh, Scotland, 2005.

[19] Benny Lehmann, Daniel Lehmann, and Noam Nisan. Combinatorial auctions with decreasing marginal utilities. Games and Economic Behavior, 55(2):270-296, May 2006.

[20] Herman B. Leonard. Elicitation of honest preferences for the assignment of individuals to positions. The Journal of Political Economy, 91(3):461-479, 1983.

[21] Andreu Mas-Colell, Michael D. Whinston, and Jerry R. Green. Microecononmic Theory. Oxford University Press, 1995.

[22] Debasis Mishra and David C. Parkes. Ascending price Vickrey auctions for general valuations. Journal of Economic Theory, 132(1):335-366, 2007.

[23] Kenneth R. Mount and Stanley Reiter. The information size of message spaces. Journal of Economic Theory, 28:1-18, 1974.

[24] Noam Nisan and Ilya Segal. The communication requirements of efficient allocations and supporting prices. Journal of Economic Theory, 129(1):192-224, 2006.

[25] David C. Parkes. Price-based information certificates for minimal-revelation combinatorial auctions. In J. Padget, D. Parkes, N. Sadeh, O. Shehory, and W. Walsh, editors, Agent-Mediated Electronic Commerce IV: Designing Mechanisms and Systems (LNAI 2531), pages 103-122. Springer-Verlag, 2002.

[26] David C. Parkes and Lyle H. Ungar. An ascending-price generalized Vickrey auction. Technical report, Harvard University, 2002.

[27] Stefan Reichelstein. Incentive compatibility and informational requirements. Journal of Economic Theory, 34:32-51, 1984.

[28] Michael H. Rothkopf, Thomas J. Teisberg, and Edward P. Kahn. Why are Vickrey auctions rare? Journal of Political Economy, 98:94-109, 1990.

[29] Lloyd S. Shapley and Martin Shubik. The assignment game I: The core. International Journal of Game Theory, 1:111-130, 1972.

[30] William Vickrey. Counterspeculation, auctions and competitive sealed tenders. Journal of Finance, 16:8-37, 1961. 